UCRL -90758

DE84 012739

ECONOMIC POTENTIAL OF INERTIAL FUSION

John H. Nuckolls

This paper was prepared for submittal to:

Fusion Power Associates Symposium on

Fusion Power Development - The Next Decade

Livermore, CA - April 2-3, 1984

April 1984

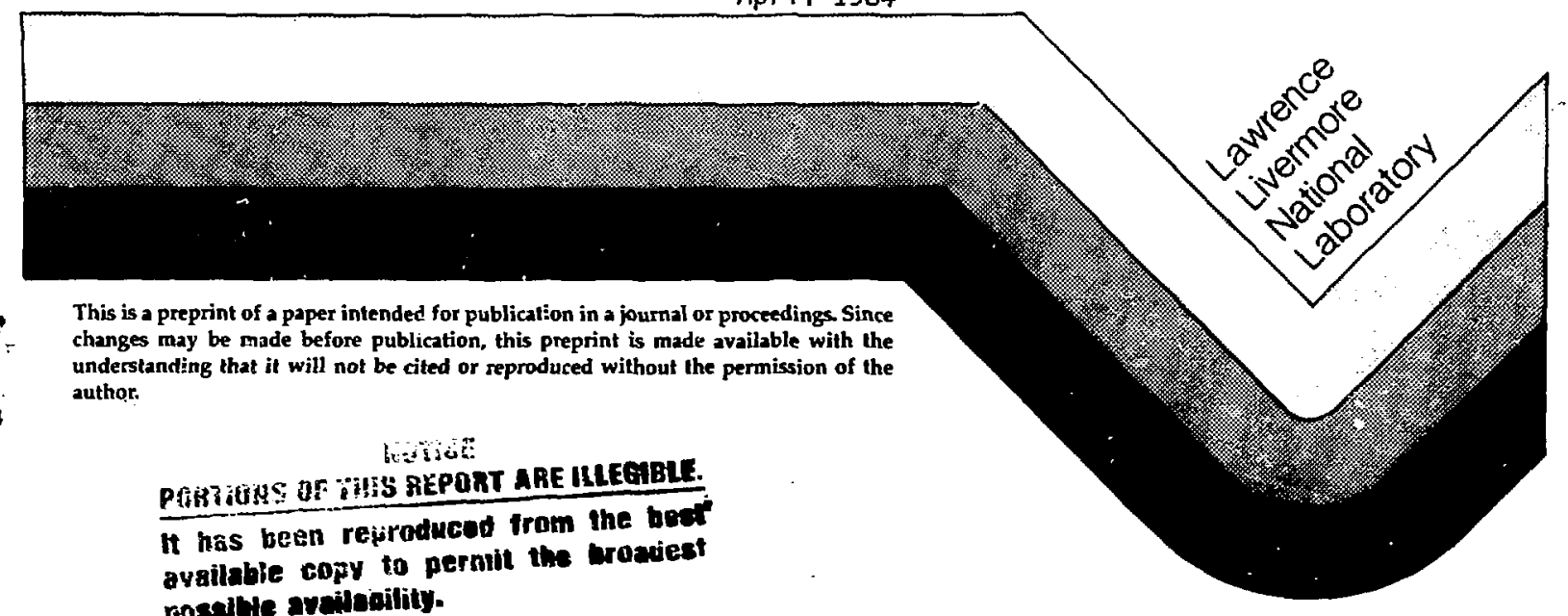
posellite avciloaility. 


\title{
Economic Potential of Inertial Fusion
}

\author{
John H. Nuckolls \\ Lawrence Livermore National Laboratory \\ April 3, 1984
}

\begin{abstract}
DISCLAIMER
This report was prepared as an account of work sponsored by an agency of the United Siates Government. Neither the United States Government nor any agency thereof, nor any of their employees, makes any warranty, express or implied, or assumes any legal liability or responsibility for the acsuracy, completeness, or usefulness of any information, apparatus, product, or process disclosed, or represents that its use would not infringe privately owned rights. Reference herein to any specific commercial product, process, or service by trade name, trademark, manufacturer, or otherwise does not necessarily constitute or imply its endorsement, recommendation, or favoring by the United States Government or any agency thereof. The viewa and opinions of authors expressed herein do not necessarily state or reflect those of the United States Government or any agency thereof.
\end{abstract}

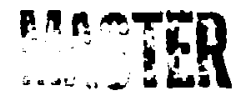


Beyond the achievement of scientific feasibility, the key question for fusion energy is: does it have the economic potential to be significantly cheaper than fission and coal energy? If fusion has this high economic petential - and I believe it does - then there are compelling commercial and geopolitical incentives to accelerate the pace of the fusion program in the near term, and to install a global fusion energy system in the long term. Without this high economic potential, fusion's success depends on the failure of all alternacives, and there is no real incentive to accelerate the program.

If my conjectures on the economic potential of inertial fusion are approximately correct, then Inerttal fusion energy's ultimate costs may be only half to two-thirds those of advanced fission and coal energy systems. Relative cost escalation is not assumed and could increase this advantage. Both magnetic and inertial approaches to fusion potentially have a two-fold economic aovantage which derives from two fundamental properties: negligible fuel costs and high quality energy which makes possible mor: efficient generation of electricity. The wining approach to fusion may excel in three areas: electrical generating efficiency, minimum material costs, and adaptability to manufacture in automated factories. The winning approach must also rate highly in enviromental potential, safety, avallability factor, lifetime, small 0 and $M$ costs, and no possibility of utility-disabling accidents. 
Econonic potential is meaningless without scientific feasibility. Most of the resources of the ICF program are now focussed on the demonstration of scientific feasibility. We have achteved high densfty in implosions ( $100 \times$ liquid density) and have demonstrated efficient target coupling with short wavelength lasers. Future key scientific feasibility experiments will be performed with woVA, PBFA II, and other facilities.

Bevond scientific feasibility, advances are required in the three key inertial fusion technologies - tagets, drivers, and reactors - in order to generate economically competitive energy. These advances appear to be feasible.

Engineering cannot be addressed experimentally with the current inertial fusion budget. However, potential engineering issues are given due weight in planning, technology development, and system analysis.

Inertial fusion has an exceptionally high environmental potential, not only for minimizing the radfoactive waste and tritium hazard, but also for eliminating the lithium hazard.

The economic potential of inertial fusion derives from time sharing of the oriver and forseeable advances in targets, drivers, and reactors. 
- Scientific Feasibility - solutions to principal sciontific problems have been partially tested. Key experiments will be performed with Nove, PBFA II, and other facilities.

- Technologies - required advances in targets, drivers, and reactors appear to be feasible.

- Engineering - not addressed experimentally with current budget.

- Environment Potential - fluld shlelding of reactor first wall reduces radioactive waste 10-100 fold. Advanced fuel burners make possible minimum radioactive waste and tritium hazard, and elimination of lithium hazard.

- Economic Potential - driver time sharing and foreseeable advances in targets, drivers, and reactors would make possible fusion onergy economically competitive with fission and coal energy, without cost escalation.

60-80-0183-0010 
For targets, the key issues are gain and the required driver energy. Severalfold improvements in these parameters are required for low cost fusion energy. These improvements appear to be feasible. With these improvements, advanced (neutron poor) fuel options would be attractive. Preliminary estimates of target costs are less than $5 \%$ of the total energy cost.

For drivers, efficiency and cost are key issues. The required improvements in these parameters for low cost fusion energy appear to be feasible. The driver requirements would be greatly relaxed by improvements in target performance, and by time sharing a single driver with several reactors. This time sharing is practical because the driver and reactor are separable (the beam can be propagated 10 meters), the driver is modular - so that it need not be shut down for repair and high rellability can be achieved, and the driver cost depends weakly on the repetition rate up to $10-20 \mathrm{HZ}$.

for low cost reactors with fluid shielded walls, rep rate and cost are key issues. The required improvements appear to be feasible.

In order to make practical fusion energy which is cheaper than coal and fission energy, it is probably necessary to exploit the high quality of fusion energy to generate electricity at very high efficiency. It is also necessary to minimize material costs, and labor costs - by developing approaches to drivers and reactors which are we 17 adapted to automated manufacture. 
Targets - Gain and driver energy are key issues. Several-fold performance improvement required for economically competitive power plant appears to be feasible. With this improvement advanced fuel options are attractive.

- Preliminary estimates of target costs are within requirements $k 10 \%$ of total energy cost).

Drivers - Efficiency and cost are key issues. Required improvements appear to be feasible. Target improvements and time sharing greatly relax driver requirements. Separability, modularity, and weak dependence of cost on rep rate make time sharing feasible.

Reactors - Fiep rate and cost are key issues in fluid shielded systems. Required improvements appear to be feasible.

Unconventional approaches which exploit the high quality of fusion energy may be required for fusion energy cheaper than coal and fission energy. Probably also require approaches to driver and reactor which are well adapted to automated factory production of transportable modules. 
There are four key environmental issues for fusion: radioactive waste, the meltdown hazard, and the lithium and tritium hazards. In inertial fusion reactors, fluid shielding may be used to reduce the radioactive waste several orders of magnitude. The shielding increases the wall lifetime iu the reactor lifetime - so that the mass of waste wall material is minimized - and reduces and softens the neutron flux so that the activation is also smalter. The shieldina also makes possible higher power. density and smaller wall area and mass. Advanced (neutron poor) fuels would also reduce the activation.

Because the wall is so much less activated, the meltdown hazard is eliminated. If the reactor is suddenly shut down, and no coolant is pumped, the first wall will not heat itself to melting by its $\therefore$ own activation.

The lithium fammibility may be eliminated by use of $\mathrm{Pb}-\mathrm{Li}$ mixtures or Li ceramic pellets. Use of advanced fuels which generate as much tritium as $\mathrm{He}^{3}$ as they consume would make possible elimination of the lithium and the tritium breeding.

For advanced fuel burning, inertial reactors have an important advantage over magnetic reactors: they have higher power densities than DT burning reactors. In inertial reactors, the power density is determined by the heat, and noutron loading of the wall and does not, as in magnetic fusion, depend on the $\overline{\sigma V}$ of the reacting fuel. 
Radioactive waste minimized
Wall lifetime $\approx$ reactor lifetime
Reduced neutron flux, softer spectrum
Fluid shielding
Reduced wall area: high power density, near spherical
Advanced fuels (neutron poor)

Meltdown hazard eliminated

Lithium, tritium hazards minimized

$\mathrm{Pb}-\mathrm{Li}$ or $\mathrm{Li}$ ceramics to eliminate flammability

Advanced fuels - eliminate lithium, tritium breeding

- Isotopically self sufficient mixtures

- Reactor power density higher than for DT fuel 
A $60-80 \mathrm{~cm}$ thick lithium blanket increases the lifetime of a common low chrome-nickel free steel chamber wall approximately 10 fold to the 30 year reactor lifetime. This is at a relatively high fusion power density of $10 \mathrm{MW} / \mathrm{M}^{2}$. The wall lifetime is more limited by atomic displacements than by helium production. The wall radioactivity is reduced 10-100 fold because of the softer and less intense neutron flux. 
THE PROTECTIVE LITHIUM BLANKET INCREASES

THE CHAMBER WALL LIFETIME

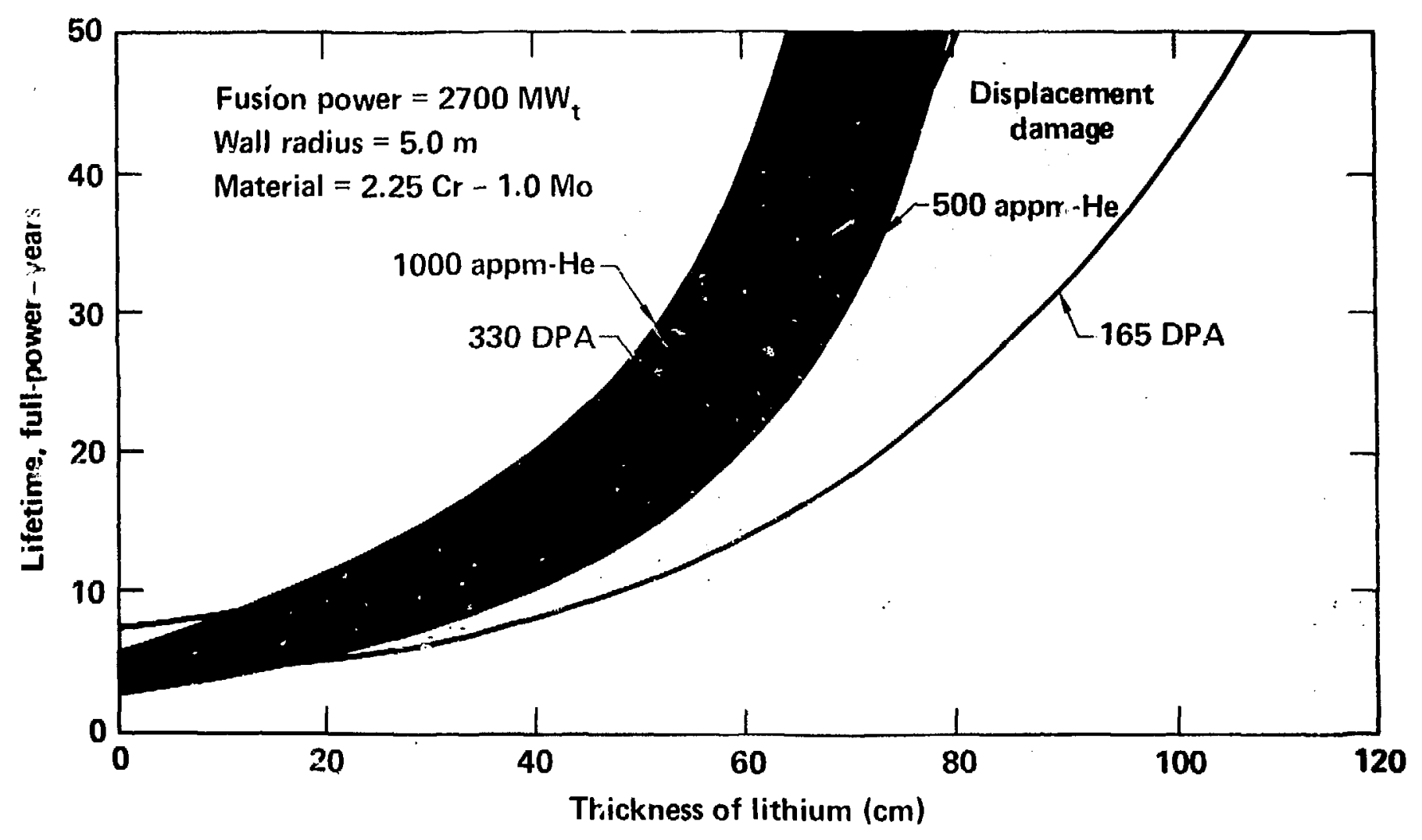

$95-01.0680-1890$ 
There are other important advantages of first wall shielding. We compare the radioactive hazards at shutdown are compared in the next figure of three different kinds of re 2 ctors, all 1000 MHe: a fission pressurized water reactor, a fusion reactor with a bare stainless steel first wall, and a HYLIFE fusion reactor with a low-chrome nickel-free steel first wall shielded by $\sim 70$ an of lithium.

All three measures of the radioactive hazard - total radicactivity, biological, and thermal decay power - show the order of magnitude advantages of the shielding. It is also clear that the bare reactor requires greatly improved first wall materia?s. Any improvement in first wall materials would, of course, also reduce the radioactivity in the fluid shielded case by the same factor. 
COMPARISON OF PWR, BARE FUSION AND HYLIFE (PER $1000 \mathrm{MW}_{\mathrm{e}}$ AT SHUTDOWN)

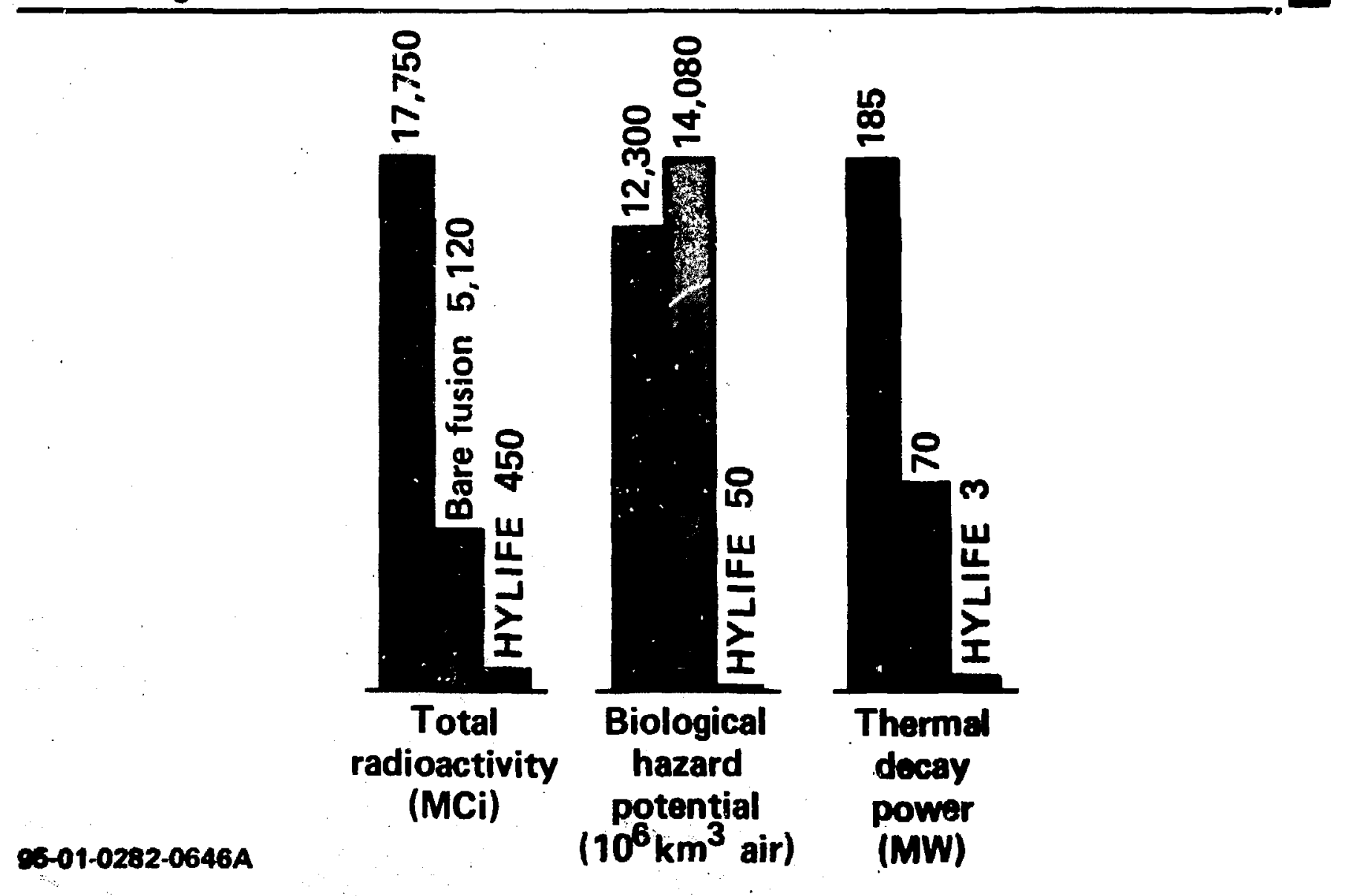


Fusion faces three important economic challenges. First, with available funds we must demonstrate scientific feasibility, achieve engineering feasibility, and build a commercially attractive demo reactor. Second, our early commercial fusion power plants should be relatively small and inexpensive (few hundred MWe and $M \$$ ) to reduce utility risks, and to achieve high learning rates. Third, the ultimate costs of fusion energy should be significantly less than those of coal and fission energy. 
Development Costs - with available funds, to demonstrats sciontific feasibility, achieve an engineering data base, and bulld a commorcially attractive demo reactor.

Commercial Learning Costs - first commercial fusion powor plants should be small (few hundred MWe/few hundred million dollars) with limited utility risks, and flexible with high technological learning rates.

Ultimate Energy Costs - at least economically competitive with advanced unescalated fission and coal energy - preferably significantly cheaper. 
This talk is mainly concerned with the ultimate economic potential of inertial fusion. An important question is, "what economic potential is necessary"? If fusion dces not have the economic potential to be cheaper than fission and coal, then several important things follow. First, there is no urgency. There are decades supply of oil and natural gas, centuries of coal and millenia of fission fucl. Seccnd, there is insufficient incentive to pay the enormous expenses of engineering, installing, and debugging a commercial fusion energy system. Here there may be a similarity to the manned-lun y. program. Recall in the decade after the successful landing of a man on the moon, the NASA budget declined. If fusion achieves scientific foasibility before having developed an economic end qame, fusion may follow in NASA's footsteps. Third, there will be little incentive to pursue the inertial backup to the mirror backup to the Tokamak, all more or less equally too expensive.

Fusion cannot depend on relative cost escalation to solve its economic problems. If fusion is feasible then the fusion/fission hybrid breeder is feasible. The hybrid limits the increase in lightwater reactor fuel costs. This limit is equivalent to a $20 \%$ increase in lightwater reactor energy costs. The cost of the hybrid is 2-3 times that of a lightwater reactor and its support ratio, that is the number of lightwater reactors of equal thermal power to that of the hybrid that it can support, is about 15 . The ratio of 3 to 15 gives the $20 \%$ factor. This estimate is relatively insensitive to large errors in hubrid cost. For example, if the hubrid costs 6 times as much as a lightwater reactor, then fission energy costs would increase an additional $25 \%$.

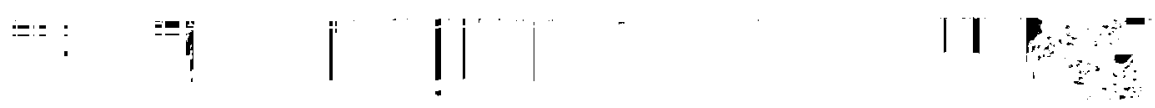


Another argument against depending on relative cost escalation is that there would be no urgency in fusion development. At $2 \%$ per year relative cost escalation, it would take 35 years to gain the factor of 2 by which fusion reactors that are now on the drawing board are inere expensive than lightwater reactors. Furthermore, the escalation arqument may have the wrong sign. Fusion involves newer and more complex technologies and is earlier on the learning curve. Fusion costs may escalate faster than fission costs. 


\section{Economic goal (requirement?)}

- If fusion does not have potential to be cheaper than fission and coal, then

- No urgency: decades of oil and natural gas, centuries of coal, millenia of fission

- Insufficient incentive to pay enormous expenses of engineering, installing, and debugging a fusion energy system

- Little incentive to pursue Inertial backup to Mirror backup to Tokamak, all more or less equally expensive'

- Fusion cannot depend on relative cost escalation

- Hybrid limit on LWR fuol costs: $20 \%$ increase in LWR energy costs

- No urgency: 35 years at $2 \% / y e a r$ to gain factor of two

- Wrong sign? Fusion involves newer and more complex technologies, earlier on learning curve 
If fusion has the potential to be cheaner than fission and coal, then the United States has a strong commercial incentive to accelerate fusion research so that U.S. Industry can be the IBM, and not the U.S. Steel, in the multi ten trillion dollar 21 st century world energy market. Other naticns have similar incentives. Furthermore, with this low cost economic potential, it would make sense to plirsue both magnetic and inertial fusion hevond scientific feasibility until the relative economic potentials are determined.

The leverage is high. It has been estimated that the total cost of developing fusion is perhaps twenty to thirty billion dollars. The cost of accelerating fusion is at most 10-20\% of this. Since operating costs are much larger than machine building costs, money will be saved by accelerating the program if an economic end game is possible. The commercial payoff is a thousand times iarger than the incremental development cost, some 10-50\% of 10-100 trillion dollars. This estimate of the 21 st century energy market is based upon the expectation that the population of the world will at least double between the 1980's and 2030's, and hopefully level off after 2030. With even very slow progress in increasing the per capita energy to-more than 2 kilowatts, the number of new reactors required will be approximately 10,000. At two billion dollars a reactor this amounts to 20 trillion dollars.

Fusion could also provide the incentive to limit coal burning and thereby to avoid the serious environmental effects of consuming the earth's remaining fossit resources. 


\section{Economic goal (requirement?) - continued}

- If fusion does have potential to be cheaper than fission and coal, then

- U.S. has strong commercial incentive to accelerate fusion development, so that U.S industry can be the IBM, not the U.S. Steel, in the multi-ten trillion dollar $\left(10^{13} \$\right) 21$ st century world energy market - other nations have similar incentives

- Fusion can prcvide an economic incentive to avoid environmental effects of coal burning, including $\mathrm{CO}_{2}$ induced global climate changes, and to avoid depletion of the worlds remaining fossil resources

Cost to accelerate fusion: $10-20 \%$ of $3 \times 10^{10} \$$

Commercial payoff: $\quad 10-50 \%$ of $10^{13}-10^{14} \$$ 
Inspection and analysis of the breakdown of the 1982 "overnight" construction costs of LWR, LMFBR, and coal power plants show that in order to be cheaper than fission and coal, fusion must reduce the cost of its reactor plant equipment severalfold to that of an LWR, and must also reduce the cost of the fusion technology equipment severalfold, ideally to less than $20 \%$ of the total power plant cost. 


\section{“Overnight" construction costs (1982\$)}

Account

\# Description

21 Structure and imp.

22 Reactor plant equip.

23 Turbine plant equip.

24 Electric plant equip.

25 Misc. plant equip.

26 Main cond. heat rej. sys.

Total direct \$/KWE

91 Construction and services

92 Home office eng., ser.

93 Field office eng., ser.

Total indirect \$/KWE
Power plant

\begin{tabular}{llll} 
LWR & LMFBR & Coal & Fusion \\
\hline
\end{tabular}

215

225

190

90

28

36

780

170

420

175

94

35

29

992

178

268

124

88

305

144

69

18

28

641

$\sim 5007$

$\sim 700$ ?

$\sim 1800$ ?

164

225

112

500

686

90

38

33

160

UE and C-ANL-821115 (Nov., '82)/J. Hovingh, Int. memo 
The 1982 cost analysis also shows a significant cost advantage for coal over fission. Part of this advantage will be erased by the costs of scrubbers. Most of fissions higher cost now appears to be due to rapidly inflating labor costs. Because fusion is newer and more complex, its labor costs may be higher still. This labor cost differential could be erased by developing approaches to fission and fusion which can best exploit standardization, modularity, and automated manufacture. 


\section{Labor accounts for most of LWR/coal cost differential}

LWR

Total "overnight" construction cost

Non-labor

Labor
$1300 / K_{E}$

$550(40-45 \%)$

$750(55-60 \%)$
Coal

$\Delta$

756*

544

$466(70 \%)$

$290(30 \%)$
84

460

Issue: Which fission and fusion approaches can best exploit standardization, modularity, and automated manufacture of transportable modules to minimize labor costs?

*Add $200 \$ / K W_{E}$ for $\mathrm{NO}_{X}$ scrubbers

Costs derived from UE and C-ANL 821115, EEDM program update (Nov. 1982) 
The crucial question is, What is the ultimate economic potential of fusion relative to fission and coal? This potencial may be estimated by considering fusion's differential assets and liabilities. Fusinn has two principal assets which could potentially confer a fact or of two advantage. First, the typical fuel cycle cost for a lightwater reactor is approximately $20 \%$ of the total husbar cost of fission energy. With the hybrid $20 \%$ escalation factor the fission fuel costs would give fusion a $40 \%$. advantage since its fuel costs are negligible. Second, there is another possible 40\% advantage which derives from a combination of two factors: the high quality of fusion enargy and the fluid insulation of fusion reactor walls. These two factors taken together make possible a $40-50 \%$ increase in the elcctrical generating efficiency. Multiplied together, fusions two $40 \%$ assets provide a potential twofold advantage over fission - if fusions liabilities can be reduced to insignificant levels.

In first generation fusion power plants which have been priced in numerous systems studies, the liabilities of fusion have been so large as to more than cancel out its cisets and result in an overall factor of two greater cost. Consequently, the critical question is, "How much can fusion's llabllities be reduced"? For inertial fusion there are two principal liabilities, first the driver and second the reactor. By reactor I mean the equivalent of the NSS or the Nuclear Steam supply sys tem. 
For the driver, inertial fusion has three options which taken together can reduce the drfver cost by almost an order of magnitude. First, since the reactor and driver are separable, it is poss fble to drive several reactors with a single driver. The triver cost increases much less than linear wfth rep rates up to 10 or 20 hertz. Second, it is estimated that foreseeable technological improvements in drivers will lead to a twofold decrease in cost. Third, improvements in targets may reduce the required driver energy and cost more than two to threefold.

Concerning the reactor nuclear steam supply system, inertial fusion reactors can approach LWR costs by utilizing fluid shielding and simple fluid energy transport systems. In addition the emergency core cooling system can be eliminated.

For both the fusion NSS and the driver, labor/fabrication costs can be minimized by developing approaches well adapted to automated manufacture of transportable modules. 


\section{Ultimate economic potential (relative to fission)}

- Fusion's assets: factor' of two advantage?

- Fuel costs $40 \%$ advantage with fission fuel cost escalation to hybrid limit (20\%)

- Energy quality $\left.\begin{array}{c}+ \\ \text { insulated wails }\end{array}\right] \longrightarrow \begin{aligned} & \text { Increase electrical generating } \\ & \text { efficleney by } 40 \%\end{aligned}$

- Fusion liabilities: $20 \%$ disadvantage?

- Technology (driver) $\longrightarrow$ Utillze time sharing, improve driver and targot?

- Reactor (NSS) — Utilize fluid shielding, simple fluid energy transport; eliminate ECCS,

- Standardized, transportable modules

(NSS: nuclear steam supply system)

$3 / 84$

$70-01-0983-3575$ 
There are several possible approaches to generating high electrical efficiency with inertial confinement reactors.

In the Pillbox MHD scheme, the idea is to explode the target in a pill-shape mass of 11 thium approximately two $14 \mathrm{MeV}$ neutron mean-free-paths thick so that most of the energy of the or burning pellet is absorbed in the pill. Because of the pill shape, most of the matter explodes axially in two jets perpendicular to the plane of symmetry of the pill. These two hot lithium jets are ejected from the reaction chamber into MHD generators where efficient electrical generation occurs. The walls of the reactor are protected by liquid lithium jets as in HYLIFE and the MHD generator is protected by a thin surface layer of liquid lithium. As the pellet yield approaches several hundred MJ, it is estimated the exploding lithium will be sufficientiy hot to provide the required electrical conductivity.

In the pebble-bed scheme, electricity is generated by conventional means. In this scheme the explosion chamber walls are protected by a slowly moving layer of solid lithium ceramic nellets (e.g., lithium-oxide). The entire chamber is rotater to hold the pellets against the wall. The pellets are neated by the $14 \mathrm{MeV}$ neutrons as well as by the $x$-rays from the fusion explosion and the hot plasma debris. The hot pebbles are circulated cutside of the main reaction chamber to a helium cooled pebble-bed heat exchanger. Very high temperature helium ( $\sim 1200^{\circ} \mathrm{K}$, is directed to a helium gas turbine, and a steam bottoming cycle. Estimated gross plant efficiency is $55 \%$ (see UCRL-89273, December 1983, John H. Pitts). 
No matter what approach is used to generate electricity it is important to minimize the recirculating power fraction. This is accompl ished by requiring that the driver have a sufficiently high efficiency, greater than 15\%, and the target have sufficiently high gain, greater than about 200 . Then with a $50 \%$ electrical generating efficiency only about $5 \%$ of the electrical energy generated by the reactor is consumed by the driver. 


\section{Possible approaches to higher electrical}

efficiency $(50-60 \%$ ?)

- Pillbox MHD scheme

- Explode target in pill shaped mass of lithium, two noutron MFP thick

- Hot lithium debris ejected axially through MHD generators

- Walls protected by lithium jets and fluid layers

- Pellet bed scheme

- Explosion chamber walls protected by thick, slowly circulating layer of lithium oxide pellets

- Hot pellets circulate to helium cooled pellet bed heat exchanger

$-1200^{\circ} \mathrm{K}$ helium to gas turbines and steam bottoming cycle.

- Minimize recirculating power fraction $(<10 \%)$

- Driver efficiency greater than $15 \%$, target gain greater than 200 
This slide shows a figure from a report by G. P. Lasche, a scientist at the Livermore Laboratory, who designed an inertial fusion MHD reactor for his Ph.D. thesis.* This reactor differs in a fundamental way from the one that I have described. It uses a large spherical ball of lithium around the pellet and attempts to generate electricity not only by the debris ejected through the classic MHD generators but al so by compression of the magnetic field by the exploding sphere of lithium. Because the HYLIFE jets are eliminated, this scheme uses an extremely large ball of lithium, some 3 meters in diameter, to control the impulsive duration. With a scaled down lithium curtain, the 3 meter ball may be replaced by a basketball size pill-shaped object. The idea of an ICF driven MHD generator merits detailed investigation.

*G.P. Lasche, "Inertial Confinement Fusion with Direct Electric Generation by Magnetic Flux Compression," UCRL-88210, Apri1 1983. 


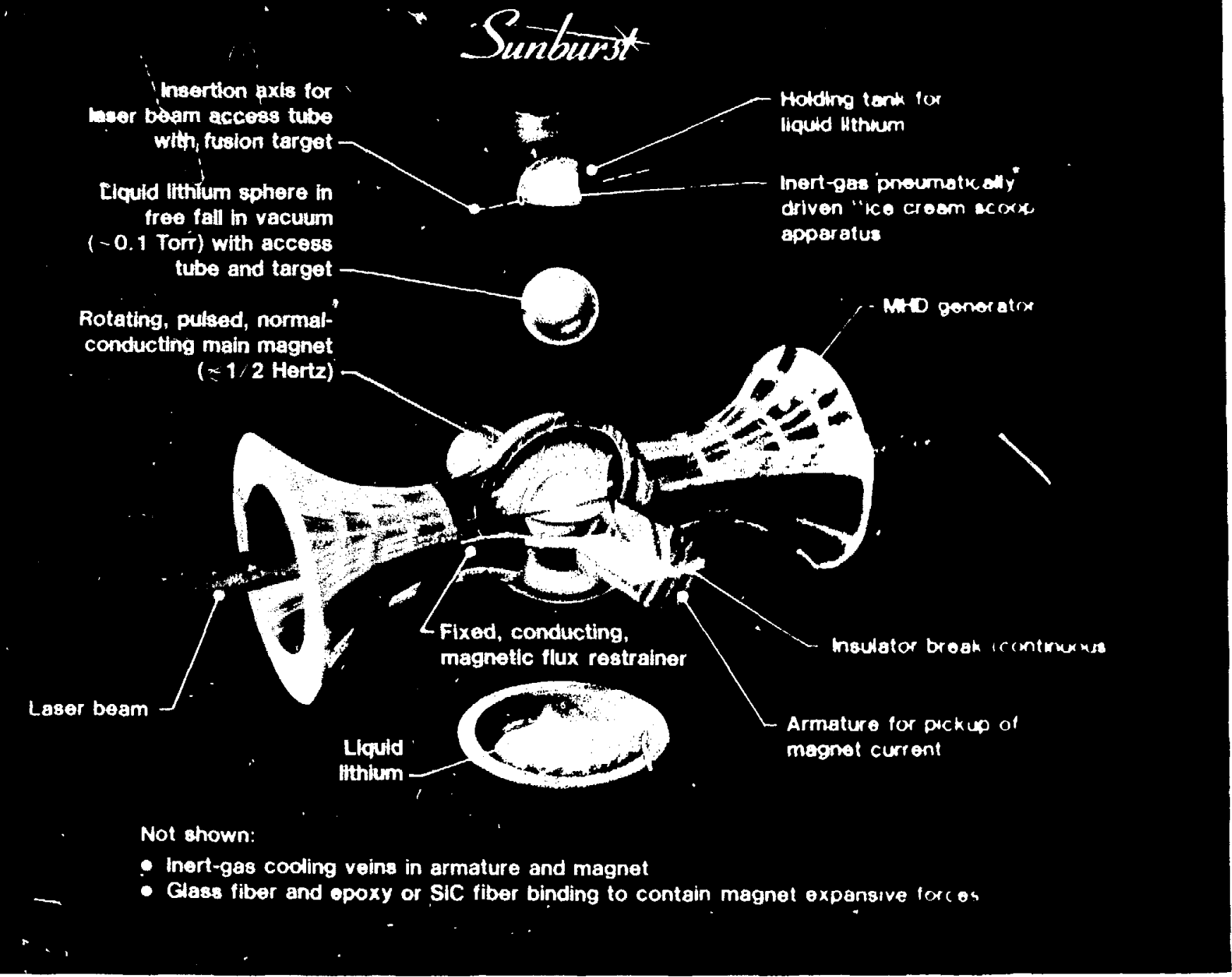


Another Livermore scientist, John Pitts, has developed the pebble-bed scheme that I have described.* In this approach the reaction chamber is rotated in order to keep the layer of pebbles against the wa11. The pellets are heated to more than $1200^{\circ} \mathrm{K}$ by fusion neutrons, $x$-rays, and plasma. Since these pellets are lithium oxide (or lithium ceramic), the lithium fire hazard is el iminated.

Relative to fission HTGRs, this scheme has the advantage that the pellets contain no fission products and may be circulated without perturbing the reactor critically. Relative to coal schemes, the high temperature material problems are less difficult with inert helium than with hydrocarbon combustion products.

*J. H. Pitts, "A Centrifugal-Action Sol id-Breeder Reaction Chamber," UCRL-88208, Apri1 1983; and "Mechanical and Thermal Design of the Cascade Reactor," UCRL-89273, December 1983. 
Cascado: A centrifugal-action, solidhthium-ceramic granule blanket reactor

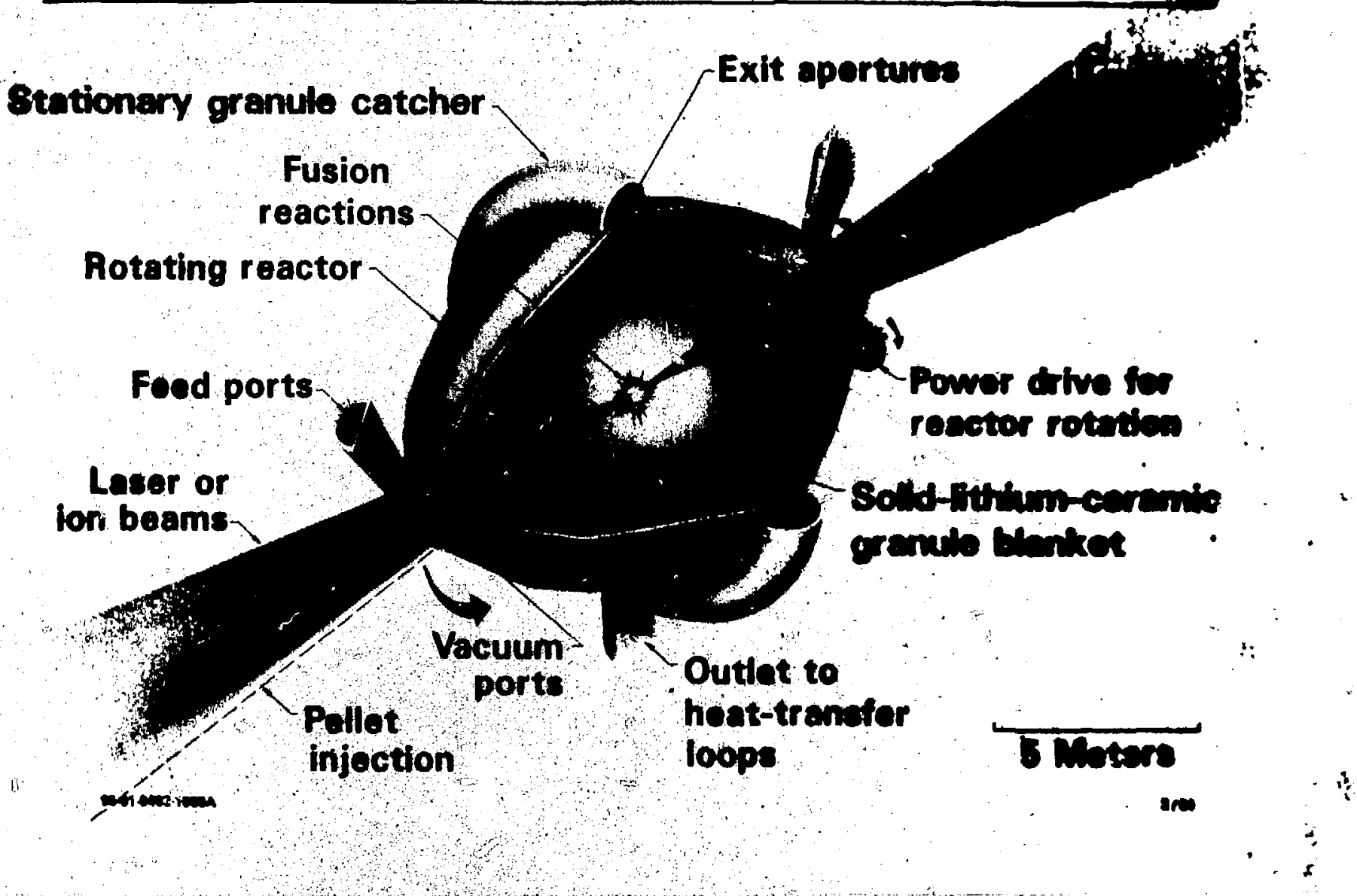


There are at least three possible approaches to reducing the driver cost. First the driver energy and cost may be reduced by improving the target performance. A recent development which could reduce the required driver energy is the use of polarized fuel. There are other means of improving the target performance which have not been described in the open Titerature.

Secondly, the driver technology is steadily being improved. Typical multimegajoule driver technologies are now costed at approximately 400/\$/J. At 2 MJ most driver experts believe that a factor of 2 improvement is possible. It is also important to develop drivers with high efficiency and with efficient target coupling.

The third approach is to timeshare a single driver with 2 to 4 reactors. The cost vs rep rate is very favorabie - only a 10-20\% increase in cost for a 10 hertz rep rate. The driver may be made sufficiently reliable by using redundancy either by adding a few extra beams to a laser or by adding modules to an accelerator. For both lasers and accelerators rapid switching and long distance beam propagation is feasible. The overall result with $200 / \$ / \mathrm{J}$ at $2 \mathrm{MJ}$ and 2 reactors is to reduce the cost of the driver to 200 million dollars per GWe reactor. Proponents argues that further reduction in driver costs are feasible. 


\section{Possible approaches to reducing driver cost}

- Reduce driver energy (improve target performance)

- Polarized fuel: $4 \mathrm{MJ} \rightarrow 1.3 \mathrm{MJ}$ at constant gain

- Other means?: increase gain several fold

- Optimize driver

- Driver technology $400 \$ / J \rightarrow 200 \$ / J$ (at $2 \mathrm{MJ}$ )

- Efficiency, target coupling

- Time share driver: 2 reactors

- Cost vs rep rate: $10-20 \%$ for $10 . \mathrm{Hz}$ or more

- Reliability: redundancy

- Rapid switching, long distance beam propagation

- Ultra cheap drivers?

Total improvement: $200 \$ / \mathrm{J} \times 2 \mathrm{MJ}, 2$ reactors $\rightarrow 200 \mathrm{M} \$ /$ reactor 
The Maxwell velocity averaged reaction rate of DT increases about $50 \%$ with optimal nuclear polarization. Preliminary theoretical calculations by Dick More, at the Livermore Laboratory, predict that this nuclearly polarized DT would survive implosion and ignition in an inertial confinement fusion pellet without depolarization (e.g., by collisions or magnetic fields). Since the dominant thermonuclear invarfant is $\rho$ r $\bar{\sigma} v$, and since the mass of a sphere is proportional to $(\rho r)^{3}$ at constant density, the driver energy is proportional to (target gain $\sqrt[\sigma v]{ })^{3}$. Therefore, for a factor of $50 \%$ increase in $\overline{\sigma v}$, the driver energy would be reduced by $1.5^{3}$ or about a factor of 3. It is possible that there is an additional improvement due to the anisotropic fusion products. However, the minimum size target and driver energy may be determined by preheat and thermal conduction.

It may be possible to construct ICF targets containing polarized DT. However, this has not yet been demonstrated. 


\section{Polarized fuel reduces driver energy $>2$ fold}

- $\overline{\sigma v} \times 1.5$ for DT with optimal nuclear polarization

- Theory predicts DT survives implosion and ignition w/o depolarization by collisions or magnetic fields

- Isotropic approximation: dominant TN invarient is $\rho R \overline{\sigma v}$

- Driver energy $\sim \frac{\text { target gain }}{3}$

- Driver energy $\times 1 / 3$ for $\overline{\sigma v} \times 1.5$ (100\% polarization)

- Additional improvement from anisotropic fusion products

- Minimum size set by preheat and thermal conduction

- Are targets with polarized DT practical? 
The inertial fusion nuclear steam supply system cost may apparently be reduced to that of an LWR. The cost of the NSS is proportional to the mass of the material involved and to the cost per unit mass. At constant power the reactor mass decreases as the power density increases. By fluid shielding the walls of the reactor so that the neutron flux is reduced $10-100$ fold, the reactor power density may be increased nearly to that of an LWR. The fluid shielding also extends the first wall lifetime to that of the reactor. This reduces the mass severalfold. In addition, the mass of the inertial fusion containment building may be substantially smaller than that of an LWR.

Fluid shielding also makes feasible the use of relatively inexpensive materials in the reactor first wal1. The mass of lithium could be minimized by the use of advanced fuels.

The other factor in cost per unit mass is fabrication, principally labor costs. This is driven by the complexity of the structure and by the assembly at the reactorsite. In most first generation fusion reactors, the fabrication cost is high because of the use of complex heat transport systems involving miles of welded tubing. In fluid shielded inertial fusion reactors, the fluid provides a simple inexpensive means of absorbing the energy of the neutrons and transporting heat to the electrical g̣enerating system. 
Possible approaches to reducing fusion NSS costs to those of second generation fission reactors

- Minimize mass

- Extend first wall lifetime

- Match fission power censity $\}$ Via fluid shielding

- Lightweight containment building: reduced pressure requiroment, no meltdown hazard

- Minimize cost/mass: avoid expensive materials

- Reactor wall: use fluid shielding and low cost alloys

- Lithium, tritium: use advanced fuels, ineroased powor density

- Minimize labor costs

- Manufacture standardized, transportable modules in automated factories

- Eliminate complex structures (e.g. miles of welded tubing) 
A fluid shielded inertial fusion reactor may approach the size and power density of an LWR. In general, fusion reactors without fluid shielding are much larger and more massive than LWRs, use more expensive materials, and require larger, more massive containment buildings. 


\section{THE HYLIFE VESSEL IS COMPARABLE IN SIZE AND POWER DENBITY TO A PRESSURIZED WATER REACTOR VESSEL OF THE SAME POWER}
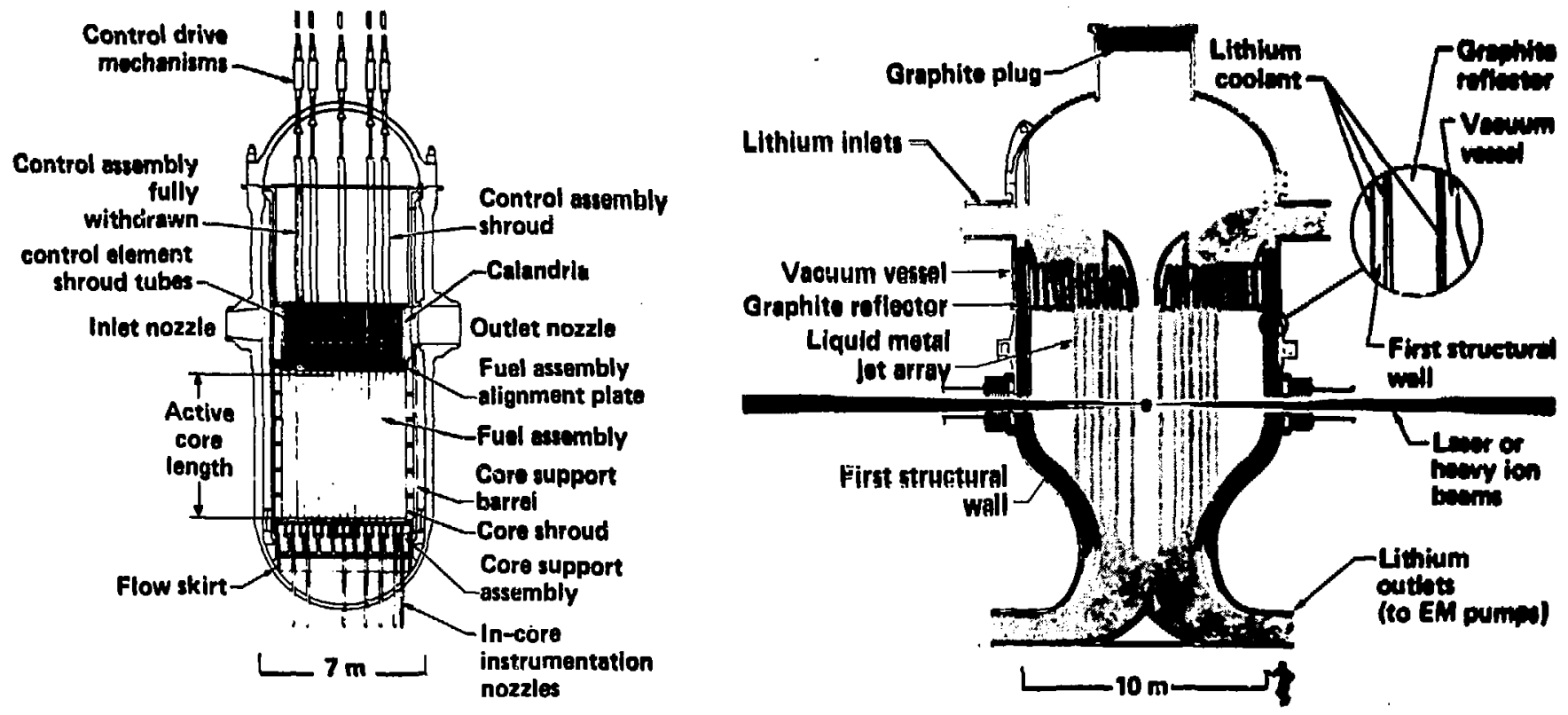

Matranom 
The bottom line is perhaps best given by a sensitivity analysis of the minimax case, that is the case where fission achieves its maximum potential. We consider a second generation one GWe LWR reactor which has zero defects, is constructed "overnight" in an automated factory, and is accepted by the public. It is hybrid fueled so that the overall cost escalation due to fuel depletion is limited to 20 percent. We compare this second generation fission power plant with an ICF power plant having an equal "overnight" capital cost. The fission capital cost is escalated $20 \%$ to account for the hybrid, and the fusion cost includes a $20 \%$ increase for the driver and target factory. The fusion and fission NSS's are assumed to have equal cost. The winner will be determined by how much electrical power the fusion fiant can produce. He compare the ratio of the busbar cost of electricity from the ICF reactor to that of the LWR. Three cases are examined. In all three cases we assume that the inertial fusion reactor achieves $55 \%$ electrical efficiency as compared to $40 \%$ for the LWR.

In case number one we assume that the inertial fusion driver, target, and nuclear steam supply system economic goals are met. In this case we find that the Inertial fusion busbar cost is only $60 \%$ of that of the optimum lightwater reactor. In the second case, we assume that the target gain is reduced twofold. In this case the inertial fusion still has a significant advantage: its busbar cost is $65 \%$ of that of the fission reactor, and its "overnight" cost is $10 \%$ higher. In the third case we assume two failures. The target 
performance is down twofold and the driver costs are twice as high as projected. In this case, inertial fusion has $80 \%$ of the corresponding fission busbar cost, and $130 \%$ of the "overnight" capital cost. 
Sensitivity analysis: ICF vs. second generation fission

\section{ICF assumptions}

(1) $55 \%$ electrical efficiency ; driver, target, NSS meet goals

(2) Same as (1), except target gain down two fold

(3) Same as (2), except driver costs twice as high

\section{Busbar} cost ratio

$0.60 *$

0.65

0.80
Capital cost ratio

$1^{t}$

1.1

1.3

* One GWe, $40 \%$ efficient second generation hybrid fueled fission reactor vs. ICF power plant with equal "overnight" construction cost.

tIncludes $1 / 15$ of hybrid capita! cost 
To estimate the net effects of these various assumptions and estimates on the projected busbar costs of fusion, fission, and coal powerplants, I have suitably madified the cost estimates in the August 1982 DOE document, "Projected Costs of Electricity from Nuclear and Coal-fired Power Plants," (DOE/EIA-0356/l). All three plants have the same thermal power, 3000 MW. Since the fusion plant has $40 \%$ higher electrical generating efficiency (55\% vs 40\%), its $1650 \mathrm{MW}$ electrical output is also $40 \%$ higher than the $1200 \mathrm{MW}$ output of the fission and coal plants. The fission plant is a single 1200 Mise system, whereas the coal plant includes a pair of 600 MWe reactors, and the fusion plant includes three 550 MWe reactors timeshared with a single one Mu driver. The fission capital cost is escalated to include the 20\% hybrid factor, and both the fission and fusion capital costs are reduced $30 \%$ for possible labor cost savings in automated manufacture. The fusion capital cost/KWe is reduced $40 \%$ below that of the fission reactor to account for the higher electrical efficiency, and increased $10 \%$ to account for the reduced size of the three components of the fusion reactor and the larger electrical generators, turbines, etc. For coal, the capital cost is increased $20 \%$ for scrubbers and the fuel cost is shown for a nominal case and for a power plant located near a coal field.

The result is a significant economic advantage for fusion relative to both fission and coal -- with the exception of a scrubberless coal plant located near a coal field. Even in this case coal has at best a $10 \%$ advantage only if fusion cannot make significant savings in labor costs. 


\section{Projected fusion, fission, coal bus-bar costs}

Levelized costs (mills/KWH) at $3000 \mathrm{MWth}$

Fusion $(55 \%)$ Fission $(40 \%)$ Coal $(40 \%)$

(3X550 MWe) (1200 MWe)

(2X600 MWo)

Capital

$O$ and $M$

Fuel
$17-21$ **

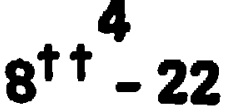

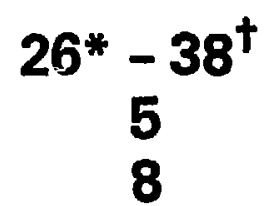

$39-51$

Total

$24-32$

$29-47$

Costs in equivalent 1980 constant \$ levelized over 30 years beginning in 1995 at average annual capacity factor of 65\% (DOE/EIA-0356/1; August '82)

* Labor costs reduced to those of coal plants (30\% of capital) * * Scrubbers (20\% of capital costs) tEscalation for Hybrid (20\% of total cost) ttFavorable location (near coal field) 
If these conjectures are correct, inertial fusion may have the economic potential to be significantly cheaper than fission and coal. This potential derives from low fuel costs, higher electrical generating efficiency, and forseeable reductions in driver costs. It is not yet clear whether inertial or magnetic fusion has the highest economic potential.

A low cost economic potential would provide strong commercial incentives to accelerate the pace of fusion development in the near term, and to install a fusion energy system in the long term. It would also provide the economic incentive necessary to avoid fossil fuel induced environmental changes, including $\mathrm{CO}_{2}$ induced global climate changes in the 21 st century.

The magnetic and inertial fusion programs should increase their programs to develop approaches with high economic potential. 
- Inertial fusion may have the economic potential to be ignificantly cheaper than fission and coal. It is not yet clear which fusion approach has the highest economic potential.

- Low cost economic potential would provide strong commercial incentives to accelerate the pace of fusion development in the near term, and to install a fusion energy system in the long term. This low cost potential would also provide the economic incentive necessary to avoid $\mathrm{CO}_{2}$ induced global climate changes in the 21st century. 